\title{
CARDIOVASCULAR RISK SCREENING SERVICES IN SUDANESE COMMUNITY PHARMACIES
}

\author{
SUMIA S MOHAMED ${ }^{1,2,3 *}$, DALIA A BALLAL ${ }^{3}$ \\ ${ }^{1}$ Department of Pharmaceutics, Faculty of Pharmacy, University of Khartoum, Sudan. ${ }^{2}$ Department of College of Pharmacy and Health \\ Sciences, Ajman University, Fujairah Campus, UAE. ${ }^{3}$ Clinical Pharmacy Program, Faculty of Pharmacy, University of Khartoum, Sudan.
} Email: msumia@hotmail.com

Received: 21 December 2018, Revised and Accepted: 09 May 2019

ABSTRACT

Objectives: This study aims to investigate the Sudanese community pharmacists knowledge and practice regarding cardiovascular disease (CVD) risk assessment services.

Methods: A non-interventional, descriptive, cross-sectional community pharmacy-based survey was conducted on 190 community pharmacies in Khartoum state.

Results: The response rate was 91\%. The obese and elderly received the highest ratings for CVD risk assessment services. Hypertension, hyperlipidemia, type 1 and type 2 diabetes mellitus, and age groups $>55$ and 36-55 years were the most targeted categories for screening. Variable responses were reported regarding the type of fasting lipid profile that is to be screened. Although most participants had the appropriate equipment for screening, only a few had cholesterol measure kits. One-third of the participants claimed to have screening evaluation forms and $27 \%$ reported the use of cardiovascular risk charts or calculators. Overwhelming majority had no documentation records and the most available reference is the BNF.

Conclusion: The current knowledge and practice of CVD risk assessment are inadequate and need fundamental development. CVD services were provided by respondents on voluntary bases without full knowledge or appropriate training on proper assessment and evaluation of the risks that they measured. This needs to change if pharmacy's potential is to be reached. Collaboration between health authorities and universities is essential to acknowledge the new roles of the pharmacist and provide the appropriate knowledge, training, and practice standards needed to promote and implement the change process that is required.

Keywords: Community pharmacy, Cardiovascular disease, Screening services, Risk assessment.

(C) 2019 The Authors. Published by Innovare Academic Sciences Pvt Ltd. This is an open access article under the CC BY license (http://creativecommons. org/licenses/by/4. 0/) DOI: http://dx.doi.org/10.22159/ajpcr.2019.v12i7.31600

\section{INTRODUCTION}

The World Health Organization figures estimate that cardiovascular disease (CVD) caused the death of 17.7 million people worldwide, being the largest cause of death. CVD is predicted to remain the number one cause of death globally in the foreseeable future. Over three-quarters of CVD deaths take place in low- and middle-income countries [1]. In the Sudan, CVD is rated third of the top 10 causes of death in hospitals and hypertension and diabetes mellitus (DM) are two of the top 10 leading diseases for hospitals admission [2]. Mukesh et al. investigated CVD prevalence and drug utilization patterns at a tertiary care hospital in Northeastern India. Their findings indicated that hyperlipidemia, hypertension, and ischemic heart disease were the most frequently diagnosed diseases and diabetes, anemia, and asthma were comorbidities associated with CVD. Male patient, 60-90 years, was most affected and admitted to the department of cardiology [3].

CVD has a substantial impact on people's health and quality of life. It also causes an increased use of health-care resources and services, representing a significant proportion of health-care expenditure. CVD risk assessment services could identify patients with poorly controlled risk factors and significantly reduce their risk for cardiovascular events. Despite the fact that CVD risk is associated with hypertension, diabetes, dyslipidemia, and smoking, these risk factors remain poorly identified and controlled. Their early identification and treatment are essential to prevent excess morbidity, mortality, and unnecessary health-care spending.

Community pharmacists are ideally placed to assist in the detection, education, and referral of individuals at elevated risk of CVD and similar conditions. The feasibility, potential benefits, and effectiveness of a community pharmacy-based CVD risk screening services are well documented. Systematic reviews, meta-analysis, and multicenter randomized controlled trials showed that pharmacies are feasible sites for such services, where a significant number of previously unknown cases of risk factors such as hypertension, hypercholesterolemia, and diabetes were identified [4-6]. Many studies demonstrated successful community pharmacist interventions in identification and targeting asymptomatic individual with high risks to develop CVD and referral to practitioners for further follow-up [7-10]. Adults in England aged 40-74 years are eligible for the assessment of CVD risk based on their body mass index, cholesterol, blood pressure, and alcohol use. People having a health check may also be tested for type 2 diabetes if the doctor thinks it is necessary. The results of the health check are given as an overall cardiovascular risk score (low, moderate, or high). Low and high risks are defined as less than $10 \%$ and more than $20 \%$ chance of a heart or circulation problem in the next 10 years. Pharmacies in the United Kingdom dispense prescriptions and other medicines, but increasingly also offer testing for common conditions, and provide advice on minor ailments. In addition, some pharmacies provide checks of blood pressure, cholesterol, and blood glucose and offer screening for chlamydia and allergies [11].

A comprehensive study project investigating the current situation of CVD risk screening services in community pharmacies in the Sudan was carried out by the authors. There were five aspects of the project; the awareness, attitude, knowledge, and practice of participating community pharmacists as well as the barriers of implementation. The awareness, attitude, and barriers were reported in a previously published article by the authors [12]. The aim of the present article 
is to address the knowledge and practice of Sudanese community pharmacists regarding CVD risk screening services.

\section{METHODS}

The study was approved by the Faculty of Pharmacy, University of Khartoum Ethical Research Committee. The methodological details of the study design, setting and population, sampling procedure, and data collection as well as piloting were described earlier in another article published by the same authors [12]. The total number of questions that are related to this article was 17 . Both closed-and open-ended questions were used. The demographic characteristics of participating community pharmacists were documented using four questions. Community pharmacists knowledge and practice of screening services were investigated using five and eight questions, respectively. At the end of the questionnaire, pharmacists were invited to express comments regarding the development of community pharmacy practice in the Sudan.

\section{Data analysis}

The responses in each section were subjected to frequency analysis using SPSS $^{\circledR}$ (version 13) for descriptive statistics. Results were expressed as frequency and percentage.

\section{RESULTS}

The questionnaire reliability was considered acceptable $(\alpha=0.7[95 \%$ confidence interval, $0.62-0.77])$. A total of 190 questionnaires were distributed. One hundred and seventy-three completed questionnaires were returned, after one to three reminders, giving a response rate of $91 \%$. The majority of participants aged $22-30$ years with B. Pharm and up to 5 years' practice experience (Table 1 ).

Fifty-nine of the 173 participants claimed to actually perform CVDs risk screening services in their pharmacies (Fig. 1). They were the focus of this article. For assessing their knowledge and practice, a number of questions were formulated to ensure that they provided the services according to standard procedures. The questions investigated the targeted candidates, risks and age groups, availability of patient evaluation forms, screening equipment, risk factor assessment tools, and references. In addition to, the methods used to evaluate CVD risk.

The obese received the highest ratings for screening followed by the elderly than the smokers (Fig. 2).

CVD risks that community pharmacists mostly screen were hypertension, hyperlipidemia, and DM (Fig. 3).

As can be shown in Fig. 4, the respondents mostly targeted only two age groups for hypertension screening. About $80 \%$ of the participants considered that blood pressure is high when the reading is $150 / 95 \mathrm{mmHg}, 74 \%$ considered it abnormal if the reading is $140 / 90 \mathrm{mmHg}$, and $5 \%$ considered it high when it is $130 / 85 \mathrm{mmHg}$.

The majority of respondents claimed to perform screening for type 1 and type 2 DM. A considerable number does not know the type of diabetes that requires screening and a small number of screens for gestational DM (GDM) (Fig. 5). About 44\% of the study participants indicated that they perform recheck for negative results of diabetes every 1 year, $6 \%$ claimed it is done every 2 years, and $22 \%$ does not know.

Variable responses were reported regarding the type of fasting lipid profile to screen as a measure of total count of lipids (Table 2).

Most of the participants had a glucometer and about one-half had sphygmomanometer and weight and height scales in their community pharmacies. Only a few had cholesterol measure kits (Fig. 6). About $34 \%$ of the participants reported the use of patient's evaluation forms.
Table 1: Demographic characteristics of respondents

\begin{tabular}{ll}
\hline Characteristics & Frequency (\%) \\
\hline Gender & \\
Male & $96(55.5)$ \\
Female & $77(44.5)$ \\
Age (years) & \\
22-30 & $127(73.4)$ \\
$31-40$ & $31(17.9)$ \\
$41-50$ & $6(3.5)$ \\
$>50$ & $9(5.2)$ \\
Qualifications & \\
B. Pharm & $170(98.3)$ \\
Pharmacy diploma & $3(1.7)$ \\
M. Pharm & 0 \\
Ph.D. & 0 \\
Practice experience (years) & \\
0-5 & $125(72.3)$ \\
6-10 & $33(19.0)$ \\
11-20 & $6(3.5)$ \\
>30 & $3(1.7)$ \\
& $6(3.5)$ \\
\hline
\end{tabular}

Table 2: Type of fasting lipid profile screened by participants

\begin{tabular}{ll}
\hline Type of lipid profile & Percentage \\
\hline Low-density lipoprotein & 33 \\
Total cholesterol & 11 \\
Triglycerides & 11 \\
High-density lipoprotein & 6 \\
None of the above-mentioned types & 56 \\
Other measures & 6 \\
\hline
\end{tabular}

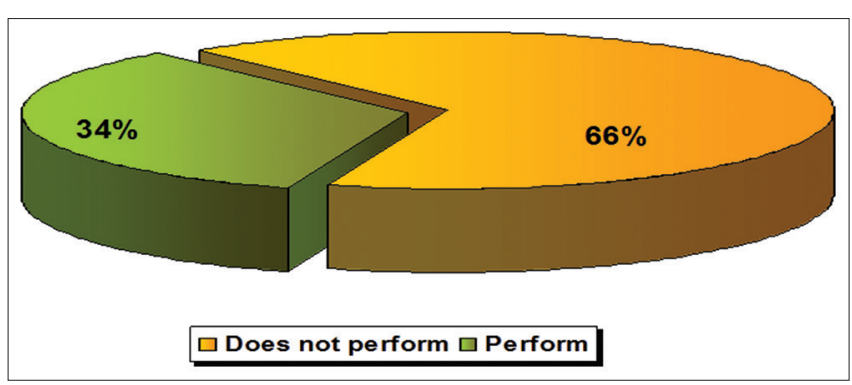

Fig. 1: Performance of cardiovascular disease screening services in respondent's pharmacies

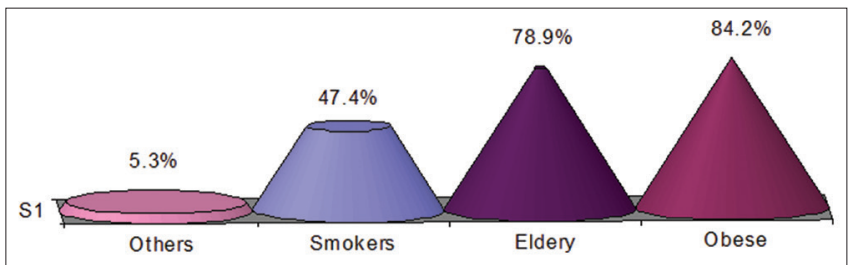

Fig. 2: Targeted customers for cardiovascular disease risk screening

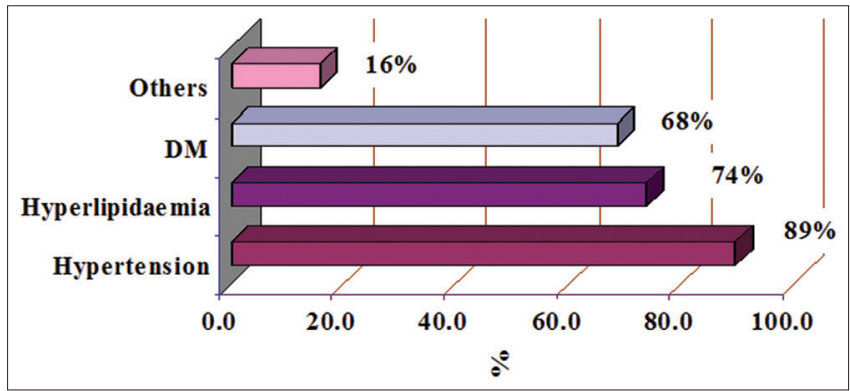

Fig. 3: Cardiovascular disease risks that necessitate screening 


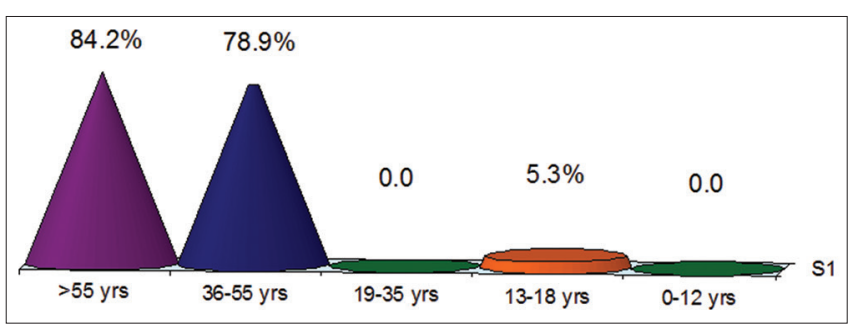

Fig. 4: Target age groups for hypertension screening

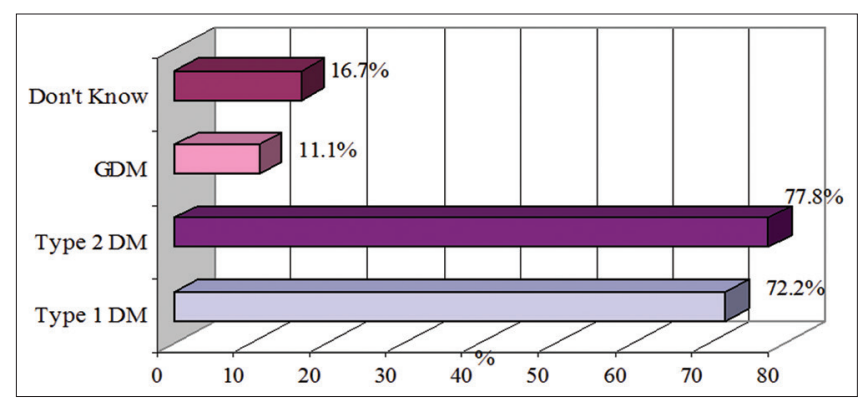

Fig. 5: Types of diabetes that necessitate screening

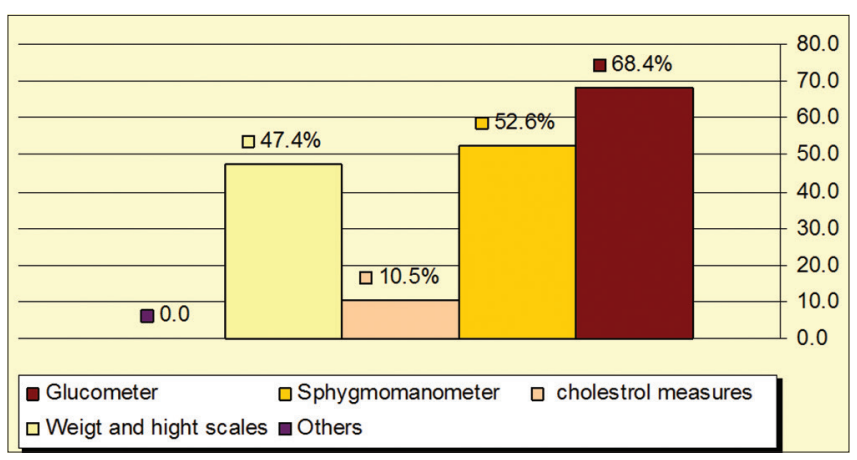

Fig. 6: Equipment available for screening purposes

Fig. 7 illustrates reference available at the surveyed community pharmacies, with the BNF being the most available one.

In response to the tools used for evaluating CVD risk, one-half of the participants did not know how to evaluate, about one-quarter do not use any evaluation tools and the rest were familiar with the CVD risk charts and calculators (Fig. 8). Overwhelming majority (94\%) has no documentation records in their pharmacies.

\section{DISCUSSION}

This study aimed to explore knowledge and practice of CVD risk screening in Sudanese community pharmacies. Although community pharmacists in many countries assumed the responsibility of implementing CVD risk assessment services, in the current study, only one-third of participants claimed to perform these services.

The majority of participants were young with a B. Pharm. A study in Australian showed that younger pharmacists and pharmacies in regional shopping centers were associated with a higher level of preventive services for CVD [13]. This indicates that patient-focused services in community pharmacies can be applicable at any educational level after graduation following appropriate knowledge and training.

The most targeted candidates mentioned by the participants for CVD screening services; the obese, elderly, and smokers give indication that they are aware of to whom the service should be offered. At least one-third of all disease burdens worldwide are caused by tobacco,

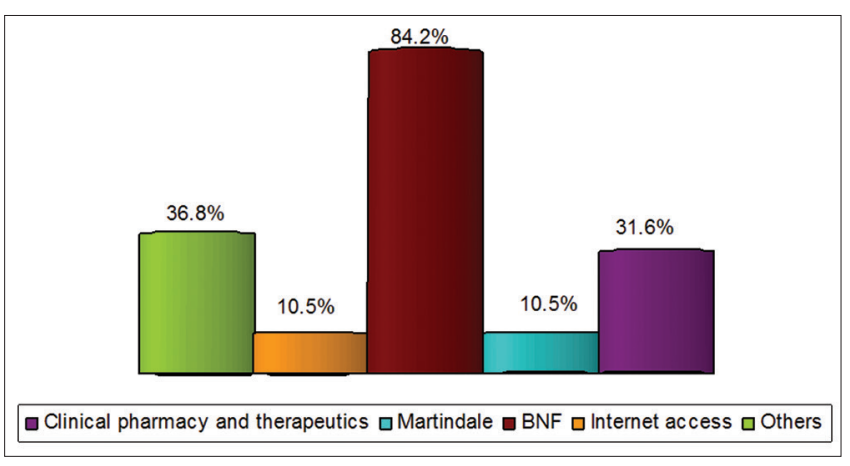

Fig. 7: References available in respondent's pharmacies

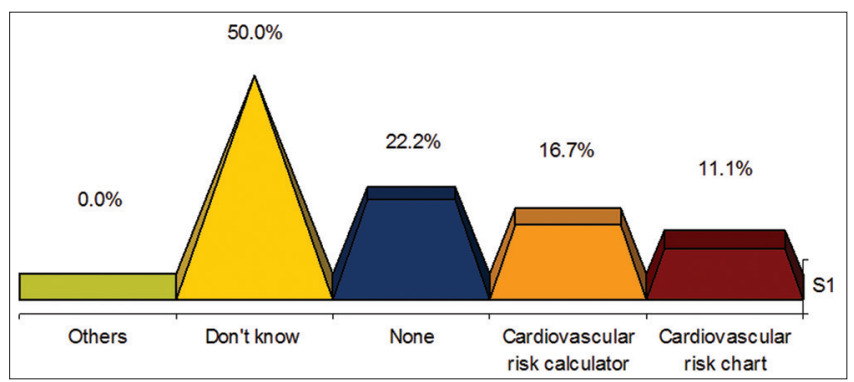

Fig. 8: Tool used for the assessment of cardiovascular disease risk

alcohol, blood pressure, cholesterol, and obesity. Furthermore, more than three-quarters of CVD results from tobacco use, high blood pressure, cholesterol, or their combination [14]. Hypertension was the most commonly reported cardiovascular risk screened by the participants. As has been mentioned earlier, in Sudan, hypertension and diabetes are two of the top 10 leading diseases for hospital admission [2]. Accordingly, community pharmacists must not ignore that comorbidity of CVD occurs with diabetes and that diabetes remains as one of the most serious risks in Sudan and should be focused on and screened as well as hypertension. Based on expert opinion, the American Diabetes Association recommends screening for type 2 DM every 3 years in all adults beginning at age 45 years. Testing should be done every 2 years starting at 10 years of age or at the onset of puberty if it occurs at a younger age. Screening for type $1 \mathrm{DM}$ is not recommended. The participants stated performance of screening for both type 2 and type $1 \mathrm{DM}$ and only a few screened GDM. Risk assessment for GDM should occur at the first prenatal visit. Women at high risk (positive family history, history of GDM, marked obesity, or member of a high-risk ethnic group) should be screened as soon as feasible [15].

The age groups targeted for hypertension screening by the majority of participants represent a positive finding since all adults 40 years onward, who have no history of CVD or diabetes, and who are not already on treatment for blood pressure or lipids, should be considered for an opportunistic comprehensive CVD risk assessment in primary care. Younger adults ( $<40$ years) with a family history of premature atherosclerotic disease should also have their cardiovascular risk factors measured [14].

Community pharmacistsare ina position to reinforce the implementation of a variety of clinical guideline treatment goals for hypertension, such as systolic $\mathrm{BP}<140 \mathrm{mmHg}$ and diastolic $\mathrm{BP}<90 \mathrm{mmHg}$, total cholesterol $<5 \mathrm{mmol} / \mathrm{L}$, and hemoglobin A1c $<7 \%[14,16,17]$. The current study found that the majority of community pharmacists are aware of the clinical guidelines of targeted blood pressure during screening. This is very significant for patient's counseling and referral. Nasution et al. showed that counseling in hypertensive patients improved adherence to prescribed medications and reduced blood pressure and the risk of CVD [18]. 
One of the main requirements for implementing screening services in community pharmacies is the availability of patient evaluation forms. In the current study, the majority of community pharmacist performed screening services without patient evaluation forms, missing one of the basic rules of practice. Some screening equipment were available to participants. For a successful screening service, all equipment must available and community pharmacists must be trained on their use, including appropriate screening and assessment techniques. The majority of participants were performing CVD risk screening services without using primary prevention methods. Therefore, they were not able take decision for estimating at-risk individuals for developing cardiovascular problems over 10 years, and hence, no referral actions or health promotion advices were given. Accordingly, pharmacist contribution to cardiovascular risk preventive screening services was self-effacing. Peterson et al. indicated that a pharmacy-based CVD risk profile screening together with appropriate educational programs has the potential to cost-effectively identify and refer many undiagnosed individuals at high risk of cardiovascular events, and help decrease the national burden of heart disease [7].

Only one-quarter of the participants reported the use of CVD risk calculators and charts. In general, the most commonly used tools for CVD risk assessment are the New Zealand Guidelines, the Sheffield Table, and the Joint British Societies' cardiovascular risk prediction charts [16]. Cardiovascular risk charts are intended to allow the introduction of the total risk stratification approach for the management of CVD $[14,16]$. Risk assessment in primary prevention is facilitated by computer software to make the estimations. However, inbuilt assumptions and default values used by particular packages need to be recognized to avoid misinterpretation. A computer-based decision support has yet to be demonstrated to affect clinical decisions or clinical outcomes. The risk charts are accessible through websites [17]. As an example, the first step in assessing a person's CVD risk is to measure the major cardiovascular risk factors; smoking habit, blood pressure, and lipids (total cholesterol and high-density lipoprotein) and then use the Joint British Societies' cardiovascular risk prediction chart (or computer program) to estimate total CVD risk according to age and gender [16]. With the current community pharmacy practice situation and the available facilities to measure CVD risks, cardiovascular risk prediction charts for primary prevention available in the BNF can be used if computer programs are not available $[14,19,20]$.

A full range of records should accompany any service provided by a pharmacist and the service should be fully auditable. Patient's details should be stored, electronically or otherwise, along with their consent forms, blood pressure, glucose, cholesterol, or other measurements together with any recommendations made. Unfortunately, it was found that overwhelming majority of participants had no records in their pharmacies. This reflects that no documentation had occurred within the current practice, which interferes with the fact that documenting the care provided in practice reflects commitment to the patient, adherence to practice standards, and the desire to learn from clinical experience [19]. Pharmacists need to continue their own education regarding CVD so that they can educate and support patients, identify patients needing medical attention, and serve as a community resource for promoting a healthy lifestyle.

\section{CONCLUSION}

CVD screening services are underprovided in community pharmacies in Khartoum state and need further development. The limited services provided, were performed on voluntary basis without appropriate knowledge and practice standards. Unfortunately, these services are not officially recognized as part of the Sudanese pharmacist responsibilities and do not represent one of the current roles of the pharmacist. Collaboration between the Ministry of Health, the Pharmaceutical and Medical Associations, and Universities for the recognition of CVD risks screening services as well as provision of knowledge and training programs for community pharmacists is essentially needed.

\section{ACKNOWLEDGMENT}

The authors appreciate the support and assistance of all participating pharmacists and community pharmacy owners. Our appreciations are extended to the pharmacists who helped in distributing and collecting the questionnaires and the Faculty of the Pharmaceutics Department for their constructive discussions and opinions.

\section{AUTHORS' CONTRIBUTIONS}

Sumia Sirelkhatim Mohamed guided and supervised the research, interpreted the results, and prepared the manuscript. Dalia Ahmed Osman Ballal carried out the survey and analyzed the results.

\section{CONFLICTS OF INTEREST}

The authors declare that they have no conflicts of interest.

\section{REFERENCES}

1. World Health Organization. Cardiovascular Diseases CVDs; 2017. Available from: http://www.who.int/news-room/fact-sheets/detail/ cardiovascular-diseases-(cvds).

2. National Ministry of Health, Directorate of Pharmacy Annual Report, Khartoum State; 2015

3. Mukesh K, Vicky D, Shruti M, Dinesh S, Neha M, Mangla L. Cardiovascular disease prevalence and drug utilization patterns at a tertiary care hospital in Northeastern India. Int J Pharm Pharm Sci 2016;8:116-9.

4. O'Donovan D, Byrne S, Sahm L. Pharmacist's use of screening tools to estimate risk of CVD: A review of the literature. Pharmacy 2014;2:27-39.

5. Willis A, Rivers P, Gray LJ, Davies M, Khunti K. The effectiveness of screening for diabetes and cardiovascular disease risk factors in a community pharmacy setting. PLoS One 2014;9:1-9.

6. Tsuyuki RT, Al Hamarneh YN, Jones CA, Hemmelgarn BR. The effectiveness of pharmacist interventions on cardiovascular risk: The multicenter randomized controlled RxEACH trial. J Am Coll Cardiol 2016;67:2846-54.

7. Peterson GM, Fitzmaurice KD, Kruup H, Jackson SL, Rasiah RL. Cardiovascular risk screening program in Australian community pharmacies. Pharm World Sci 2010;32:373-80.

8. Rohla M, Haberfeld H, Sinzinger H, Kritz H, Tscharre M, Freynhofer MK, et al. Systematic screening for cardiovascular risk at pharmacies. Open Heart 2016;3:1-7.

9. Mc Namara KP, Peterson GM, Hughes J, Krass I, Versace V, Clark RA, et al. Cardiovascular disease risk assessment in Australian community pharmacy. Heart Lung Circ 2017;26:667-76.

10. Jahangard-Rafsanjani $Z$, Hakimzadeh N, Sarayani A, Najafi S, Heidari K, Javadi MR, et al. A community pharmacy-based cardiovascular risk screening service implemented in iran. Pharm Pract (Granada) 2017;15:1-6.

11. National Institute for Health and Care Excellence. Pharmacy-Based Screening for Cardiovascular Risk; 2015.Available from: https://www. evidence.nhs.uk/about-us/publications?utm source=NewZapp\&utm medium=email\&utm_campaign=Eyes on Evidence email campaign.

12. Mohamed SS, Ballal DA. Awareness and attitude of khartoum state community pharmacists towards cardiovascular disease screening services. World J Pharm Res 2018;7:1196-209.

13. Joyce A, Berbatis CG, Sunderland VB, Dhaliwal SS. Analysis of primary prevention services for cardiovascular disease in Australia's community pharmacies. Aust N Z J Public Health 2007;31:516-9.

14. WHO Pocket Guidelines for Assessment and Management of Cardiovascular Risk: (WHO/ISH Cardiovascular Risk Prediction Charts for the African Region). Geneva: World Health Organization; 2007. p. 1-30.

15. DiPro JT, Talbert RL, Yee GC, Matzke GR, Wells BG, Posey LM, editors. Diabetes Mellitus Pharmacotherapy: A Pathophysiologic Approach. $10^{\text {th }}$ ed. New York: AccessPharmacy. McGraw-Hill Medical; 2017. p. 1143-90.

16. Hall RJ, Lefroy D, Simpson I MP. JBS2: Joint British societies' guidelines on prevention of cardiovascular disease in clinical practice. Heart 2005;91:v-1-52.

17. McGlynn S, Reid F, McAnaw J, Chinwong S, Hudson S. Pharmaceutical care: Coronary heart disease. Pharm J 2000;265:194-205.

18. Nasution A, Khairunnisa, Tanjung HR. Impacts of counseling on adherence to prescribed medications and blood pressure of hypertensive 
patients in four Indonesian primary health centers. Int $\mathrm{J}$ Pharm Pharm Sci 2015;7:114-7.

19. Royal Pharmaceutical Society of Great Britain (RPSGB). Expanded practice guidance on blood pressure monitoring. Pharm J
$2003 ; 270: 171$

20. Reamy BV, Williams PM, Kuckel DP. Prevention of cardiovascular disease: Guidelines for assessment and management of cardiovascular risk. Prim Care Clin Off Pract 2018;45:25-44 